\title{
Fallopian Tube Duplication: A Rare Anomalistic Case Report with Review of Literature
}

\author{
Priyadarshini. D* and Arathi. C.A \\ Department of Pathology, Shridevi Institute of medical sciences and research Hospital, Lingapura, Tumakuru, Karnataka, India
}

\begin{abstract}
Fallopian tube duplication is a rare Mullerian duct anomaly which is seldom reported in available literature studies. We present a rare case of unilateral duplication of fallopian tube in a 36 year old female during routine grossing of hysterectomy specimen. Microscopic examination revealed normal histology of bilateral fallopian tubes along with the accessory Fallopian tube on one side. Fallopian tube duplication may contribute for infertility, ectopic pregnancy and can be a cause for failure of sterilisation
\end{abstract}

\section{Keywords: Fallopian Tube, Duplication, Anomaly, Sterilisation}

\section{Introduction}

Fallopian tube is attached to the uterus which acts as a channel from which ovum is carried to the uterus once it is released from the ovary. Duplication of fallopian tube is a rare congenital mullerian duct anomaly which can be missed because its existence suspicion is very low. Mullerian duct anomalies are commonly reported in literatures but isolated Fallopian tube anomalies are seldom reported in research studies.

This duplication of Fallopian tube is an arena for cysts, hydrosalphinx, pyosalphinx, and also torsion. The ovum released by the ovary may land up in the accessory tube causing infertility or ectopic pregnancy. Diverse anatomic variations in fallopian tube may be noted during assessment of infertility. The patients presenting with infertility and Pelvic inflammatory disease should be compulsorily screened to rule out accessory fallopian tube.

\section{Case Report}

we present a case of Unilateral fallopian tube duplication during routine histopathology grossing in a 36 year old female patient who had undergone abdominal hysterectomy for the history of menorrhagia.

An ultrasound scan report showed an irregular cyst measuring $8.1 \mathrm{X} 5.2 \mathrm{cms}$ and small hemorrhagic cysts in left ovary. Intraoperative findings was a right ovarian chocolate cyst.

Our case was an incidental finding, while grossing the hysterectomy specimen, two separate lumens were identified on one side (Fig 1) and it was entire length. The hysterectomy specimen with bilateral adnexa was grossed, routine histopathological tissue processing was done and submitted for subsequent microscopic examination

\section{Discussion}

Mullerian duct anomalies are commonly reported in literatures but isolated Fallopian tube anomalies are seldom reported in research studies. Sanchez J et.al (3) stated that in English medical literature, accessory fallopian tubes have been rarely reported. The incidence rate documented ranged about $6 \%$ in a general population to as voluminous as $13 \%$ in women seeking infertility treatment.

Entire length replication of the uterine tubes and partial duplication of the tube is rarely observed. These accessory tubes are possibly due to a redundancy of the invagination from which infundibulum is formed. Malposition of the uterus and ovary are commonly encountered in distinctive positions but fallopian tube aberrations and faulty developmental anomalies are traceable to impaired framework of the mullerian ducts. This anomaly can be diagnosed on ultrasound, hysterosalpingogram and on histopathology sections.

The duplication of fallopian tubes may lead to infertility and ectopic pregnancy which can lead to life threatening complications (2), it can be identified commonly on hysterosalphingography in infertility cases. It is important to diagnose and document this rare congenital anomaly as, it can be a cause for failure of sterilisation procedure after postpartum and can lead to medico legal litigation issues.

Narayanan R et.al (1) reported a case of 25 year old female with inability to conceive after 6 years of married life, where hysterosalpingogram revealed this rarely observed anomaly of partial fallopian tube duplication and also highlighted that evaluation of anatomy of female genital tract is helpful for the investigation of infertility. Gandhi K.R et al (2) described a rare anomalous case report of the 


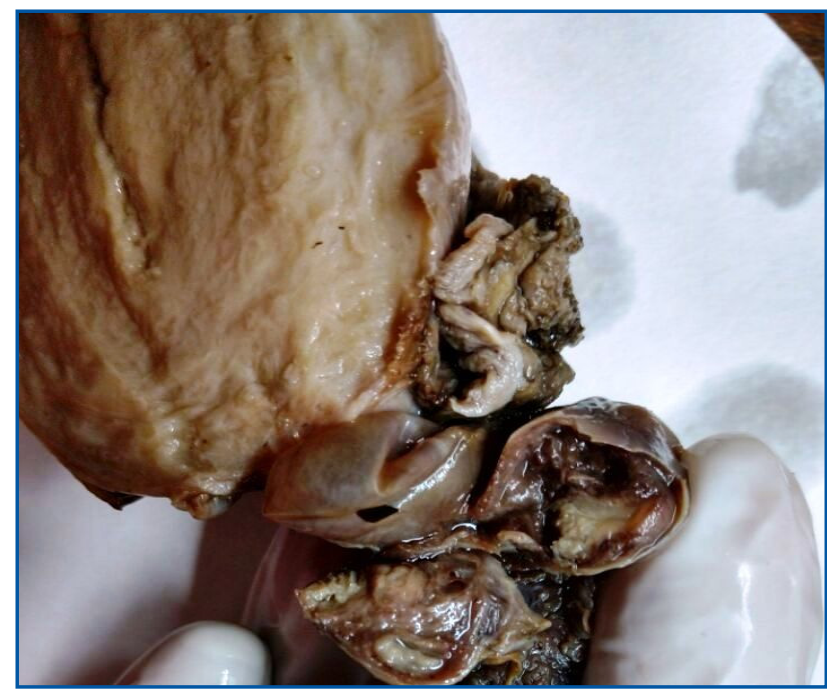

Fig. 1 : while grossing the hysterectomy specimen, two separate lumens were identified on one side and it was entire length.

accessory fallopian tube on right side from a 34 year old female cadaver during routine dissection for undergraduate students and also described that the tube was attached to ampullary portion of the main fallopian tube and was tortuous. They also reported that accessory fallopian tube can be one of the important contributory factors of infertility and also an arena for pyosalpinx, hydrosalphinx, cystic swelling and torsion.

Rottenstreich. M et.al (5) reported a rare case of accessory fallopian tube in a 16 year old virgin girl presented with history of abdominal tenderness, on laparoscopic examination revealed an accessory fallopian tube with torsion. Our case was an incidental finding of unilateral duplication of fallopian tubes with normal uterine histology.

\section{Conclusion}

Duplication of Fallopian tube is a rare mullerian duct congenital anomaly which can be missed often because of its low suspicion index. Fallopian tube duplication can form an arena for pyosalpinx, may contribute for infertility, ectopic pregnancy and cause for failure of sterilisation

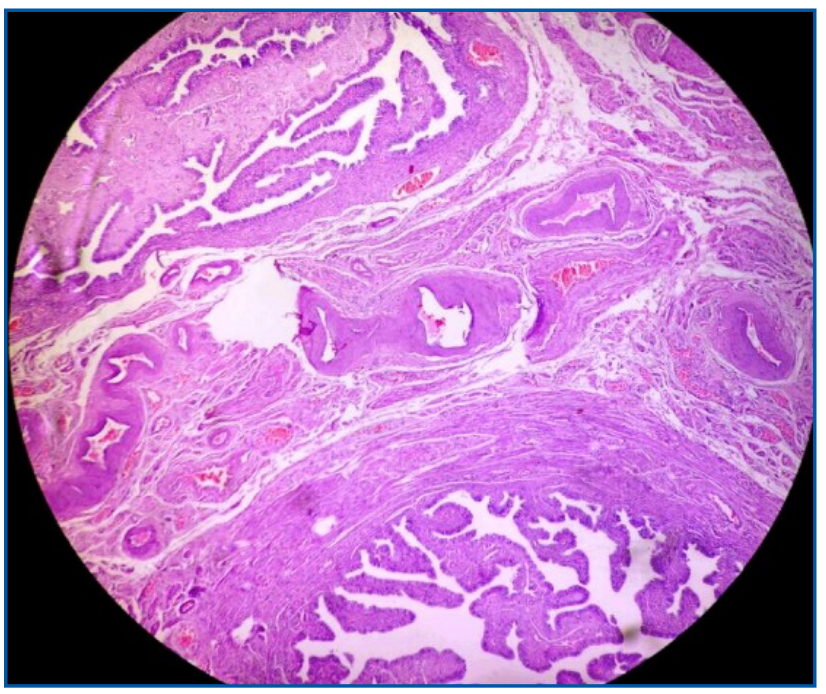

Fig. 2 : Haematoxylin and eosin stain 10X : Microscopic examination revealed two separate lumens with normal fallopian tube histology.

\section{Acknowledgements}

I thank our Medical Director, Dr Raman M Hulinaykar and our Beloved Principal Dr S D Desai for their constant support and encouragement

\section{Reference}

1. Narayanan R and Rajeev MA, duplication of fallopian tube, J Hum Reprod sci: Issue 1 vol 1, jan-jun 2008

2. Gandhi K.R, Siddiqui U.A, Wabale R.N, Daimie S.R. The accessory fallopian tube: A rare anomaly. Journal of Human Reproductive Sciences. Volume 5, Issue 3: Sep - Dec 2012; 293

3. Sanchez J, Bercaw J, Dietrich J, Accessory Fallopian Tube in an Adolescent Female: A Case Report : Journal of paediatrics and adolescent Gynaecology. April 2009 Volume 22, Issue 2, Pages 27-28

4. Shulman L.P, Elias.S. Developmental abnormalities of the female reproductive tract: Pathogenesis and nosology, Journal of paediatrics and adolescent Gynaecology. 1988 Volume 1, Issue 4, Pages 230-238

5. Rottenstreich M, Smorgick N, Pansky M, Vaknin $Z$, Isolated torsion of Accessory Fallopian Tube in a Young Adolescent: Journal of paediatrics and adolescent Gynaecology. August 2016: Volume 29, Issue 4, Pages 57-58

*Corresponding author:

Dr. Priyadarshini. D, \#108, A Block, Sai Gangotri Apartments, Ullal main road, Opp Bharath Petroleum, Muneshwaranagar, Bengaluru - 560056, India Phone: +91 7829765527

Email: priyadarshinid.arun@gmail.com

Financial or other Competing Interests: None.

Date of Submission : 11.12.2017

Date of Acceptance : 20.12.2017 Date of Publication : 31.12.2017 